\title{
Analysis of double-step response to an interplanetary shock in the dayside magnetosphere
}

\author{
K. Andréeová ${ }^{1}$, L. Juusola ${ }^{2}$, E. K. J. Kilpua ${ }^{1}$, and H. E. J. Koskinen ${ }^{1,2}$ \\ ${ }^{1}$ Department of Physics, P.O. Box 64, University of Helsinki, Helsinki, Finland \\ ${ }^{2}$ Finnish Meteorological Institute, P.O. Box 503, Helsinki, Finland \\ Correspondence to: K. Andréeová (katerina.andreeova@helsinki.fi)
}

Received: 4 March 2014 - Revised: 9 September 2014 - Accepted: 18 September 2014 - Published: 22 October 2014

\begin{abstract}
We present an analysis of double-step magnetic field enhancement caused by interplanetary (IP) shock impacts on the Earth's magnetosphere. The structures were observed by the GOES-8, 10, 11, and 12 spacecraft in the dayside geostationary orbit, particularly during northward interplanetary magnetic field (IMF) conditions. The double-step structures, similar to what is observed in the ground horizontal magnetic field $(H)$ component at low and mid latitudes, were observed preferentially on the dayside. Structures observed around 12-15 magnetic local time (MLT) displayed the steepest initial enhancement step, followed by a magnetic field strength decrease before the second enhancement step. At other dayside MLTs of the geostationary orbit, the initial response was smoother, and no decrease was observed before the second step. We suggest that this MLT asymmetry in the decrease of the total magnetic field is caused by the pushing of the plasmaspheric ions over the geostationary orbit due to the magnetospheric compression.
\end{abstract}

Keywords. Interplanetary physics (interplanetary shocks) - magnetospheric physics (plasma waves and instabilities; solar-wind-magnetosphere interactions)

\section{Introduction}

The impact of a fast forward interplanetary (IP) shock leads to significant changes in the near-Earth space environment. Abrupt changes in solar wind plasma and magnetic field parameters at the IP shock result in significant variations in the magnetospheric magnetic fields and current systems, and in the plasma convection patterns in the ionosphere and magnetosphere. In particular, the steep increase of the dynamic pressure at the IP shock (Spreiter and Stahara, 1994; Grib et al., 1979; Grib, 1982; Zhuang et al., 1981) compresses the magnetosphere on a global scale. Such compressions are manifested as positive increases of the ground horizontal magnetic field $(H)$ component at low latitudes (SI: sudden impulse) and increases in the magnetospheric fields in the geostationary orbit (Andréeová et al., 2008).

The variations of the $H$ component (Araki, 1994) depend strongly on the latitude and local time (LT). In higher latitudes during SIs, the $H$ component usually consists of two pulses with opposite polarities. Positive to negative (negative to positive) $H$ variation occurs at morning (afternoon) stations. In lower latitudes, the behavior of the $H$ component becomes more step-like. In the geostationary orbit, magnetic field increases have a double-step structure (Andréeová et al., 2011). Samsonov et al. (2007) used the global BATS-R-US MHD (magnetohydrodynamic) simulation to simulate the interaction of an interplanetary shock with the Earth's magnetosphere. The authors discussed the propagation of a fast wave through the Earth's magnetosphere and its reflection from the inner boundary, such as the plasmapause. However, it is currently not clear how the magnetic field increases at high latitudes and in the geostationary orbit are related, and, in particular, how geostationary field variations depend on the magnetic local time (MLT) and shock properties.

The analysis of the magnetospheric response to IP shocks is a complex issue. Before arriving at the magnetopause, the IP shock interacts with the Earth's bow shock and propagates through the magnetosheath. This IP shock-bow shock interaction may cause significant modification of the structure and orientation of the IP shock and even generation of new discontinuities in the magnetosheath (Prech et al., 2008). Those structures cause variations of the magnetic field and, consequently, effects on the magnetopause (Fairfield et al., 
1990). The response is also expected to depend strongly on the bow shock properties, in particular whether the region of interest is behind quasi-parallel or perpendicular bow shock. This property of the bow shock correlates with the interplanetary magnetic field (IMF) cone angle. Both upstream and downstream sides of the parallel foreshock are associated with significant wave activity; for example, ultra-low frequency (ULF) plasma waves. The ULF waves generated by wave-particle interactions at the foreshock are Pc3 waves and have relatively low amplitudes and a typical time period of about $\sim 10-45 \mathrm{~s}$. The ULF waves may affect the overall magnetic field profiles and characteristics observed in the magnetosheath, in the magnetosphere and on ground. Zhang et al. (2010) showed that the ULF activity can also be excited due to the solar wind dynamic pressure pulses impacting with the Earth's magnetosphere.

ULF waves that affect magnetospheric magnetic fields are also generated within the magnetosphere. Continuous geomagnetic ULF waves with a period of about $\sim 0.2-10 \mathrm{~s}$ are called Pc 1-2 pulsations (Anderson et al., 1992b, a). Those pulsations are generated by the electromagnetic ion cyclotron (EMIC) instability near the magnetic equatorial plane. EMIC waves are generated by temperature anisotropies $\left(T_{\perp}>T_{\|}\right)$ of magnetospheric ions in the energy range of about 10$100 \mathrm{keV}$; i.e., typical to ring current and plasma sheet energies. Yue et al. (2010) showed dawn-dusk asymmetry of the plasma temperature anisotropy, strongest around the noon sector.

In addition, the expansion of the plasmasphere to the geostationary orbit may cause changes to magnetic fields in that region and thus lead to obvious MLT asymmetries (Chappell, 1972). Elphic et al. (1996) have shown that, during high geomagnetic activity, the density of the plasmaspheric cold ions peak around 14 MLT in the geostationary orbit as a result of the compression of the magnetosphere and increase of the magnetospheric convection. Zhang et al. (2012) investigated the magnetopause response to an IP shock using observations from the THEMIS spacecraft. They observed the plasmaspheric bulge passage across the THEMIS spacecraft.

In this study, we investigate the double step structures within the Earth's magnetosphere in the geostationary orbit observed by the GOES spacecraft, as well by THEMIS on the flanks of the dayside magnetosphere. The aim of this study is to investigate the MLT dependence of double-step structures, and the role of the bow shock configuration, IP shock properties, plasmaspheric intrusion and EMIC waves in determining the characteristics of double-steps. In addition, we will compare the geostationary orbit observations to ground observations. The paper is organized as follows: the data are introduced in Sect. 2. Two cases are presented in Sect. 3. The geostationary vs. low-latitude ionospheric observations and statistical study results are shown in Sect. 4. The interpretation and summary are given in Sects. 5 and 6.

\section{Data sources}

IP shocks were selected from the ACE and Wind solar wind data, requiring one of the GOES spacecraft to be located in the dayside magnetosphere. We have used high-resolution (0.512 s) data from GOES 8, 10,11, and 12. Our study covers two periods: 2001-2002 near solar cycle 23 maximum, and 2007-2008, representing the extended solar minimum between cycles 23 and 24 . The total number of events is 24 and Table 1 summarizes the basic solar wind IP shock properties.

We have used simultaneous plasma and magnetic field data from Cluster (Balogh et al., 1997; Reme et al., 1997), GOES (Singer et al., 1996), LANL (McComas et al., 1993), THEMIS (McFadden et al., 2008; Angelopoulos, 2008), Wind (Lepping et al., 1995; Ogilvie et al., 1995; Lin et al., 1995), and ACE (Smith et al., 1998; McComas et al., 1998). The spacecraft provided data in the solar wind and in the magnetosphere. To analyze the shock response on the ground, we have used SuperMAG (http://supermag.jhuapl. edu/index.html) magnetometer observations at $1 \mathrm{~min}$ resolutions and THEMIS ground magnetometer data (http:// THEMIS.igpp.ucla.edu/) at $0.5 \mathrm{~s}$ resolutions.

\section{Case study}

In this section, we describe in detail the magnetospheric response for two events that we selected from the set of 24 shocks listed in Table 1. During event no. 1, there was very good spacecraft coverage in the dayside magnetosphere, while event no. 2 demonstrated very clear double-step structure.

\subsection{Event no. 1}

The Wind spacecraft observed the IP shock on 19 November 2007 at 17:22 UT with $20 \mathrm{~min}$ lasting quasi-radial IMF (IMF cone angle about 30 degrees) before the IP shock arrival. The IP shock front consisted of a single steep increase of all solar wind parameters: the magnetic field, the solar wind speed, the thermal speed, and the solar wind plasma density. The source of the IP shock was a magnetic cloud with a north-south polarity. The magnetosonic speed upstream from the IP shock was estimated to be about $56 \mathrm{~km} \mathrm{~s}^{-1}$ and the IP shock normal was estimated to be using minimum variance analysis about $(-0.95,0.21,0.22)$ with the IP shock speed at about $454 \mathrm{~km} \mathrm{~s}^{-1}$. The density compressional ratio, ratio between the downstream and upstream values, of the IP shock was 1.9 and the magnetic compressional ratio was 1.7 , with northward IMF upstream and downstream of the IP shock.

During quasi-radial IMF, the bow shock was quasi-parallel near the nose of the Earth's magnetosphere. Thus, a foreshock region was formed upstream of the bow shock, which probably acted as the source of ULF waves. A wavelet 
Table 1. Table summarizes 24 events, time in UT observed by the ACE, Wind and GOES spacecraft, and the solar wind IP shock parameters such as IP shock normal estimated from the minimum variance analysis (MVA), and from the magnetic coplanarity (MC) method, magnetosonic speed in $\mathrm{km} \mathrm{s}^{-1}$ and shock speed in the solar wind frame in $\mathrm{km} \mathrm{s}^{-1}$. The numbers written in bold are the cases which were shown in the paper in more detail (Sects. 3.1, 3.2, Fig. 4).

\begin{tabular}{|c|c|c|c|c|c|c|c|c|}
\hline Year & Month & Day & $\begin{array}{l}\text { ACE } \\
(\mathrm{UT})\end{array}$ & $\begin{array}{l}\text { Wind } \\
\text { (UT) }\end{array}$ & $\begin{array}{l}\text { GOES } \\
\text { (UT) }\end{array}$ & Normal vector & $\begin{array}{c}\mathrm{vms} \\
{\left[\mathrm{km} \mathrm{s}^{-1}\right]}\end{array}$ & {$\left[\begin{array}{r}\text { vsh } \\
{\left[\mathrm{km} \mathrm{s}^{-1}\right]}\end{array}\right.$} \\
\hline 2001 & 2 & 12 & $20: 45$ & $21: 31$ & $21: 30$ & $-0.94,0.33,0.10$ & 66 & 358 \\
\hline 2001 & 10 & 8 & $12: 21$ & 13:05 & $13: 10$ & $-0.87,-0.45,-0.19$ & 76 & 665 \\
\hline 2001 & 10 & 11 & $16: 20$ & $16: 50$ & 17:00 & $-0.998,0.04,0.04$ & 71 & 553 \\
\hline 2001 & 11 & 19 & $17: 35$ & $18: 15$ & $18: 15$ & $-0.99,-0.04,0.11$ & 76 & 583 \\
\hline 2001 & 11 & 30 & $17: 28$ & $18: 15$ & $18: 20$ & $-0.95,-0.31,-0.07$ & 58 & 591 \\
\hline 2001 & 12 & 30 & $19: 32$ & 20:05 & 20:05 & $-0.94,0.08,-0.34$ & 103 & 801 \\
\hline 2002 & 4 & 17 & $10: 21$ & 11:01 & 11:00 & $-0.91,-0.27,-0.31$ & 60 & 784 \\
\hline 2002 & 4 & 19 & $20: 50$ & $21: 43$ & $20: 50$ & $-0.90,-0.35,-0.27$ & 103 & 810 \\
\hline 2002 & 7 & 17 & $15: 26$ & $15: 55$ & $16: 05$ & $-0.96,0.29,-0.04$ & 65 & 405 \\
\hline 2002 & 7 & 19 & $14: 50$ & $15: 15$ & $15: 10$ & $-0.73,0.69,-0.03$ & 161 & 419 \\
\hline 2002 & 7 & 25 & $12: 59$ & $13: 29$ & $13: 40$ & $-0.90,-0.36,-0.25$ & 111 & 866 \\
\hline 2002 & 7 & 29 & $12: 40$ & $13: 15$ & $13: 20$ & $-0.94,0.17,-0.29$ & 57 & 579 \\
\hline 2002 & 11 & 9 & 16:48 & $17: 24$ & 17:50 & $-0.996,-0.02,-0.08$ & 53 & 427 \\
\hline 2002 & 11 & 9 & $17: 54$ & 18:27 & $18: 49$ & $-0.59,0.18,-0.79$ & 58 & 487 \\
\hline 2007 & 9 & 20 & 09:05 & 09:26 & $10: 10$ & $-0.46,-0.53,0.71$ & 57 & 346 \\
\hline 2007 & 9 & 27 & $10: 52$ & $10: 52$ & $11: 50$ & $-0.49,0.74,-0.46$ & 47 & 400 \\
\hline 2007 & 10 & 25 & $10: 36$ & $10: 43$ & $11: 35$ & $-0.78,-0.63,-0.03$ & 47 & 605 \\
\hline 2007 & 11 & 12 & 21:25 & 21:28 & $22: 20$ & $-0.86,0.07,0.51$ & 48 & 651 \\
\hline 2007 & 11 & 19 & $17: 15$ & $17: 22$ & 18:10 & $-0.99,0.16,0.04$ & 56 & 454 \\
\hline 2007 & 12 & 17 & 02:04 & $01: 53$ & $02: 54$ & $-0.52,0.36,-0.77$ & 54 & 406 \\
\hline 2008 & 4 & 30 & $14: 58$ & $15: 02$ & $15: 56$ & $-0.81,0.58,0.08$ & 52 & 377 \\
\hline 2008 & 5 & 28 & $01: 27$ & $01: 18$ & $02: 25$ & $-0.87,0.44,-0.24$ & 49 & 276 \\
\hline 2008 & 8 & 8 & $22: 52$ & $23: 24$ & $23: 44$ & $-0.96,-0.27,0.1$ & 90 & 247 \\
\hline 2008 & 11 & 24 & $22: 48$ & $22: 29$ & $23: 50$ & $-0.88,-0.44,-0.15$ & 48 & 502 \\
\hline
\end{tabular}

analysis (analysis not shown) of GOES 10, 11, and 12 (at 14.4, 9.3, and 13.3 MLT) magnetic field data (Fig. 1) shows that ULF activity had already been observed in the magnetosphere before the incoming IP shock response with approximately a $30 \mathrm{~s}$ period and relatively low amplitude $(\sim 1.5 \mathrm{nT})$. However the ULF wave activity was not observed by THEMIS D or E (data not shown), which were located on the morning flank of the dayside magnetosphere.

At 18:10 UT, all spacecraft in the dayside magnetosphere observed a double-step structure except for the Cluster spacecraft, located in the plasmasphere at that time. Measurements of the total magnetic field $\left(B_{\mathrm{t}}[\mathrm{nT}]\right)$ and proton density $\left(n_{\mathrm{p}}\right.$ $\left.\left[\mathrm{cm}^{-3}\right]\right)$ from the Cluster-2 spacecraft are shown in the bottom two panels of Fig. 1. The double-step increase of the $z$ component of the magnetic field is related to the magnetopause current enhancements caused by the magnetospheric compression by the IP shock. The local asymmetric decrease of the total magnetic field was probably caused by the presence of plasmaspheric ions (see the last panel of Fig. 1) with a peak around 13.5 MLT. During the magnetic field depression, the LANL-94 spacecraft, located close to GOES10 (LANL-94 at 14.6 MLT and GOES-10 at 14.4 MLT), revealed an increase of high-energy ions with the energies typical to the plasmasphere (from a few $\mathrm{keV}$ to $200 \mathrm{keV}$ ). Also an analysis of the thermal anisotropy (see Fig. 2), defined as $A=T_{\perp} / T_{\|}-1$, shows an increase $\left(T_{\perp}>T_{\|}\right)$, related to the EMIC waves.

\subsection{Event no. 2}

The Wind spacecraft observed the IP shock on 12 November 2007 at 21:28 UT with an almost Parker spiral IMF (IMF cone angle about 50 degrees). The IP shock front consisted of a single steep increase of all solar wind parameters, as in the first case. The magnetic field was mostly northward and highly variable upstream the IP shock. The magnetosonic speed upstream the IP shock was estimated to be about $48 \mathrm{~km} \mathrm{~s}^{-1}$ and the IP shock normal using minimum variance analysis (about $-0.86,0.07,0.51$ ) with the IP shock speed at about $651 \mathrm{~km} \mathrm{~s}^{-1}$. Compressional ratio was 1.4 and magnetic compressional ratio was 2.0.

At 22:20 UT, the GOES-11 spacecraft observed doublestep structure with a significant steep enhancement of the total magnetic field followed by the total magnetic field decrease before the second magnetic field enhancement, similar to what was discussed by Andréeová (2009); see Fig. 3. The 


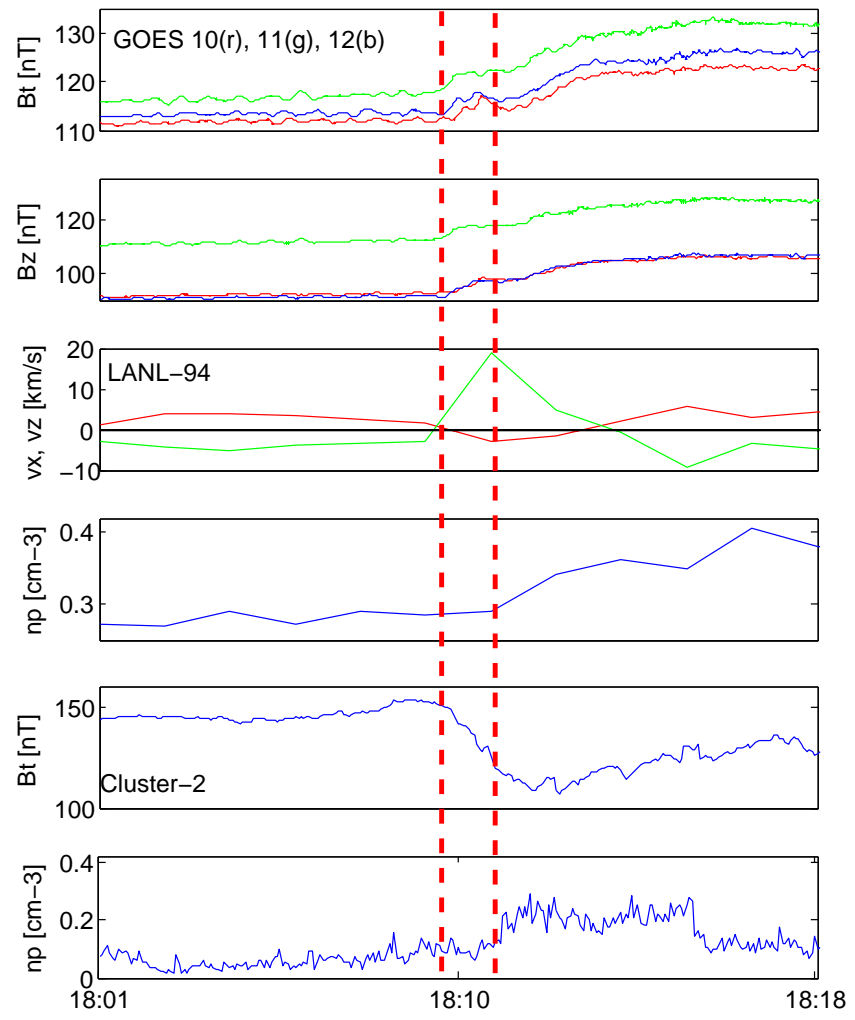

Figure 1. 19 November 2007: the first and second panels show the GOES-10, 11, and 12 (at 14.4, 9.3, and 13.3 MLT) total magnetic field and $z$ component of the magnetic field $B_{\mathrm{t}}[\mathrm{nT}]$ and $B_{z}[\mathrm{nT}]$ in the GSM (Geocentric Solar Magnetospheric) coordinate system. The third and fourth panels show LANL-1994 observation of the velocity $v_{x}$ (the red line), $v_{z}$ (the green line) $\left[\mathrm{km} \mathrm{s}^{-1}\right]$ and the partial density of high energy ions $\left(0.13-45 \mathrm{keV} \mathrm{e}^{-1}\right) n_{\mathrm{p}}\left[\mathrm{cm}^{-3}\right]$. The penultimate panel shows Cluster- 2 total magnetic field $B_{\mathrm{t}}[\mathrm{nT}]$. The last panel shows Cluster- 4 proton density $n_{\mathrm{p}}\left[\mathrm{cm}^{-3}\right]$. The first red dashed line represents the time of the first compression and the second red dashed line depicts the time of the magnetic field decrease, as observed by GOES-10 and 12 .

magnetic field properties were similar to the previous event. The decrease of the magnetic field observed by the GOES-11 spacecraft occurred around 13.25 MLT. The response profile is discussed in the following sections.

\section{Low-latitude ground observation}

Low and mid latitude ( $<60$ degrees) ground measurements from SuperMAG show certain similarities with the observations in the geostationary orbit (see Fig. 4). An incoming compression was observed $\sim 30 \mathrm{~s}$ after the GOES spacecraft observed the magnetic field intensification around 13.5 MLT in the geostationary orbit. The strongest variations are highlighted by red ovals and red arrows pointing at the MLT interval.

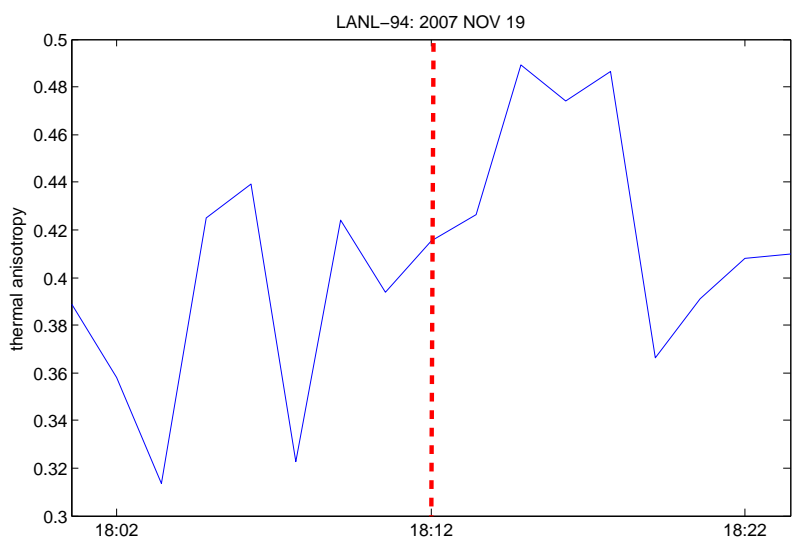

Figure 2. 19 November 2007: LANL-94 observations of the thermal anisotropy $A=T_{\perp} / T_{\|}-1$. The red dashed line depicts the time at which the GOES-10 observed the decrease in the total magnetic field.
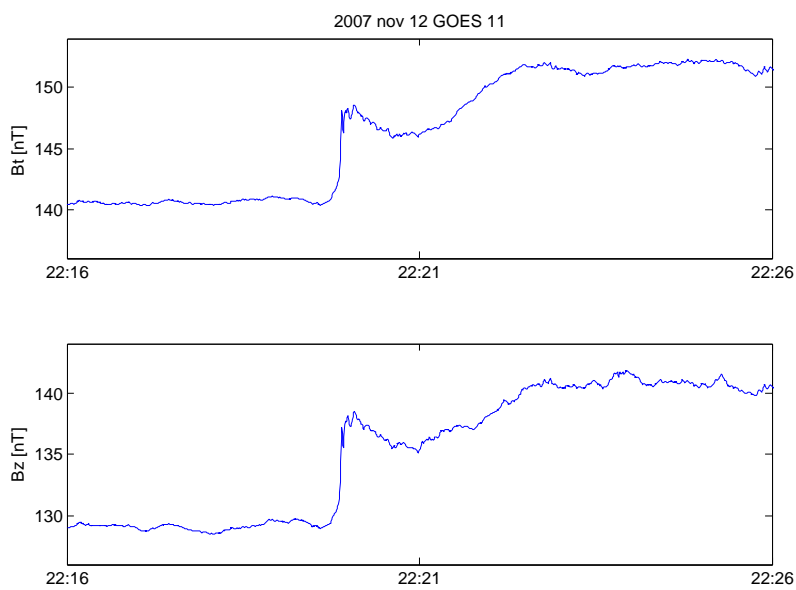

Figure 3. 12 November 2007: GOES-11 observations at 13.4 MLT: the total magnetic field $B_{\mathrm{t}}[\mathrm{nT}]$ and $z$ component of the magnetic field $B_{z}[\mathrm{nT}]$ in the GSM coordinate system.

The ground horizontal component $(H)$ has a double-step profile (Event no. 1; see Fig. 5) in lower latitudes, with a longer duration $(\sim 3 \mathrm{~min})$ compared to the GOES spacecraft observation. The higher-latitude magnetometer at Inuvik (INUV) recorded a bipolar signature in the $H$ component. The $H$ component at low and mid latitude reveals fluctuations with an average period of about $\sim 35 \mathrm{~s}$ and relatively low amplitude $(\sim 1 \mathrm{nT})$, similar to the GOES spacecraft observations. The amplitude of the decrease after the first increase was of the same order of magnitude $(\sim 1 \mathrm{nT})$. The THEMIS ground measurements were limited on the location around 8-10 MLT, due to the missing data in the other locations.

However, the connection between the geostationary orbit and the ground observations is not so clear in the case of a stronger IP shock. When the solar wind magnetic field 

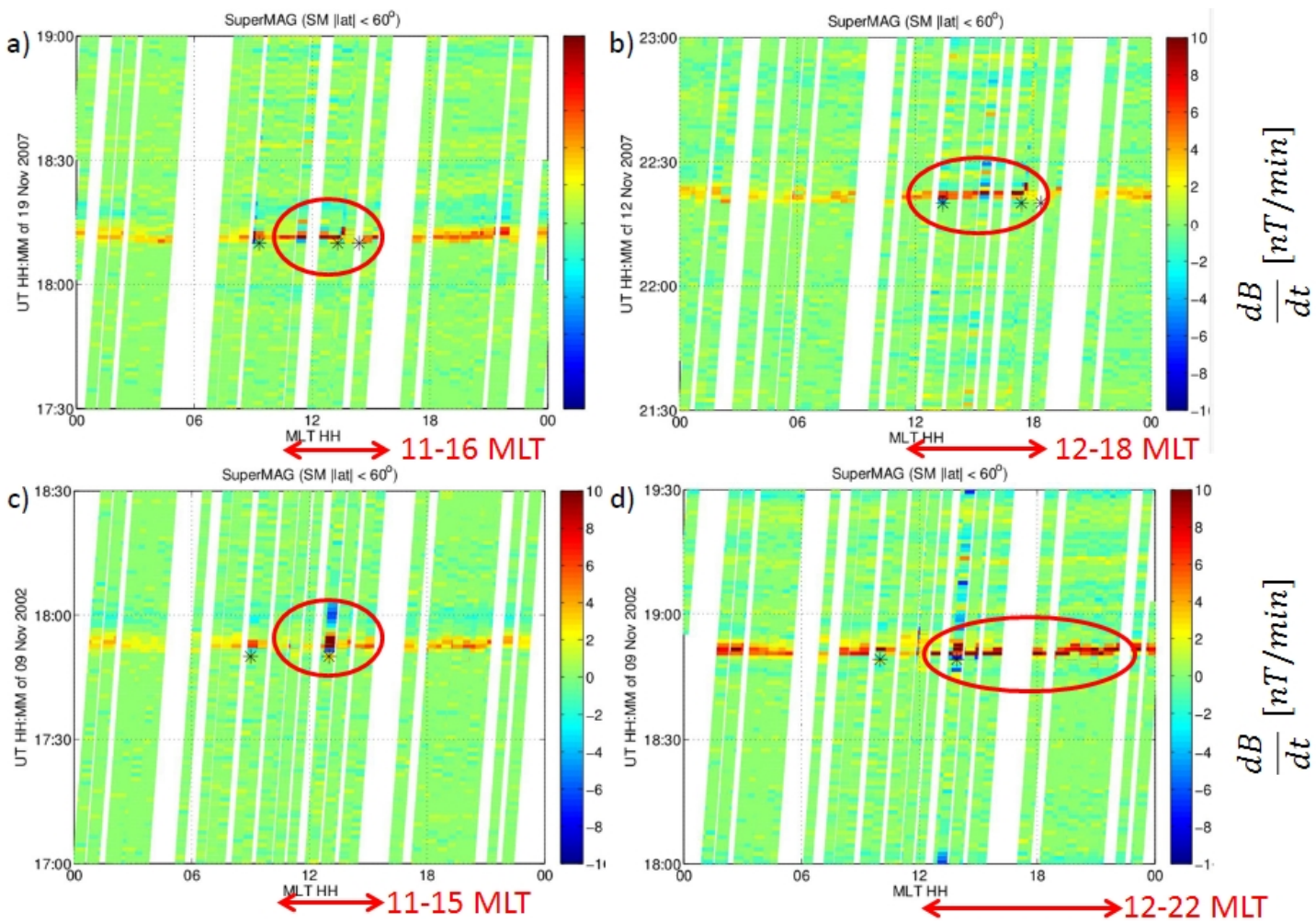

Figure 4. Low and mid latitude ( $<60$ degrees) ground observation of the time variation of the magnetic field (in $\mathrm{nT}^{-1} \mathrm{~min}^{-1}$ ) from SuperMAG data. The black stars denote MLT of the GOES spacecraft in the geostationary orbit. The upper left picture (a) represents event 1: 19 November 2007. The upper right picture (b) represents event 2: 12 November 2007. The lower two pictures (c, d) illustrate the event investigated in our previous study (Andréeová, 2009): 9 November 2002 at 17:50 and 18:49 UT. Red ovals highlight the intensification areas, whereas red arrows show the time interval in MLT.
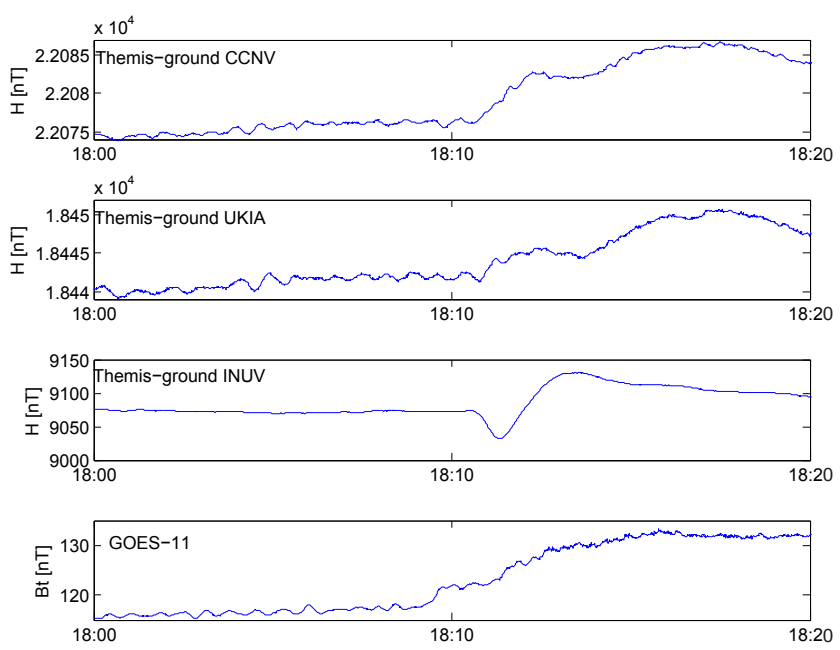

Figure 5. Example of the observations from the low-latitude ground-based magnetometer, 19 November 2007: CCNV (Carson City, geog. lat 39.2, geog. long 240.2, MLT 8.5), UKIA (Ukiah, geog. lat 45.1, geog. long 241.07, MLT 8.25), INUV (Inuvik, geog. lat 68.3, geog. long 226.7, MLT 10.2, and GOES-11 spacecraft in MLT 9.3.) compression was over 1.9 and the solar wind density compression was over 2.0, the low and mid latitude time variations of the total magnetic field were stronger over all MLTs. SuperMAG data resolution is 1 minute, which is below the resolution of the fine structure observed in the geostationary orbit.

\section{Statistical analysis}

The following analysis is based on 24 events (see Table 1) of IP shocks and high time-resolution data from the GOES spacecraft in the Earth's magnetosphere, between years 2001 and 2002 during solar maximum, and between years 2007 and 2008 during the solar minimum.

The double-step structure observed in the Earth's magnetosphere strongly depends on the location of the observation in the dayside magnetosphere (see Fig. 6) and on the IMF direction (see Fig. 7). During the quasi-radial IMF, nearly the entire bow shock is quasi-parallel and ULF waves could be launched into the magnetosphere, leading to the modification of the magnetospheric response to the IP shock impact.

The very steep initial enhancement of the total magnetic field in the GOES high-resolution data was followed by a 


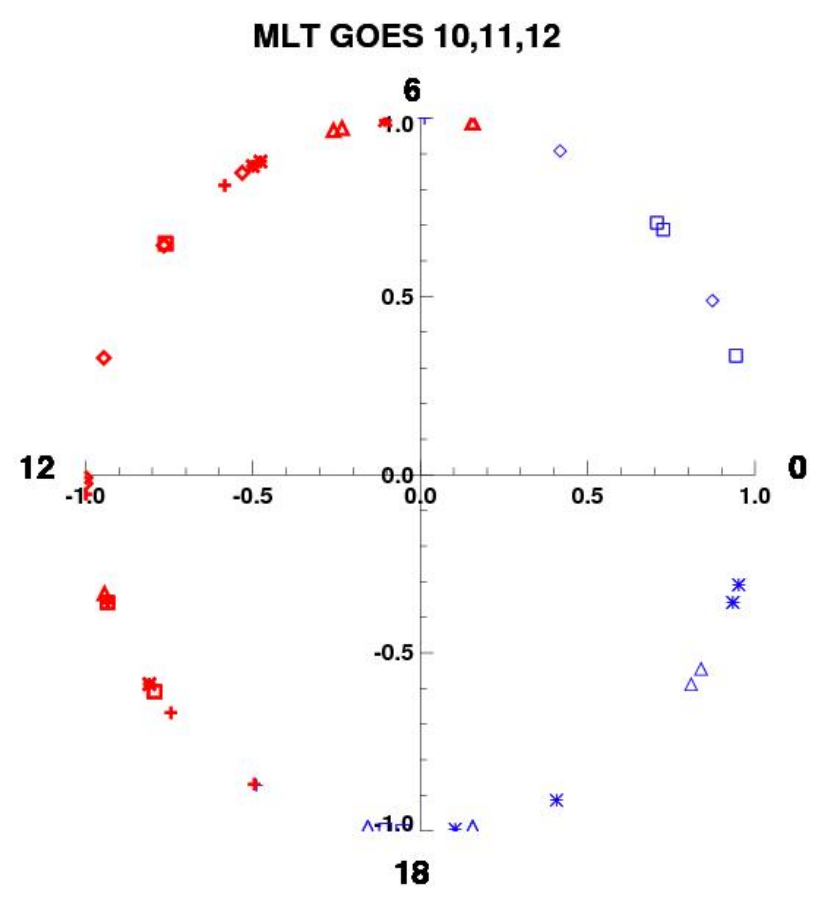

Figure 6. GOES spacecraft observations of the double-step structure in MLT coordinates. The red bold marks represent double-step structure, the blue lighter marks represent observations without a double-step structure, or even any compression. The different marks represent particular GOES 8 (star), 10 (square, diamond), 11 (plus), and 12 (triangle) spacecraft.

decrease of the total magnetic field, before the second enhancement of the total magnetic field. This structure was observed, on average, around 13.5 MLT, from 12 to 15 MLT. In the dayside magnetosphere from 5 to 12 MLT, the GOES spacecraft also observed a certain level of the double-step structure of the magnetic field, but without any magnetic field decrease between the enhancements. This behavior is very similar to that which was observed in the lower latitudes in the $H$ component (Fig. 5). There were no double-step structures observed on the evening side from 15 to 18 MLT. This sector was covered by only three measurements. Figure 8 summarizes the expected profiles on the geostationary orbit, highlighting the steepening of the double-step structure towards the afternoon sector around 13.5 MLT. There is a very weak observation of the double-step structure between 5 to 8 MLT, as also seen in Fig. 7. There are only three cases positively identified as double-step structure between 15 to 18 MLT. However, the two points are highly unclear after 17 MLT.

During the quasi-radial IMF, the response in the magnetosphere was affected by the ULF waves with a $30 \mathrm{~s}$ period, leading to the smoothing of the initial magnetic field enhancement. The overall magnetospheric compression was similar to the regular compression caused by the similar dynamic pressure and magnetic field compression in the solar wind.
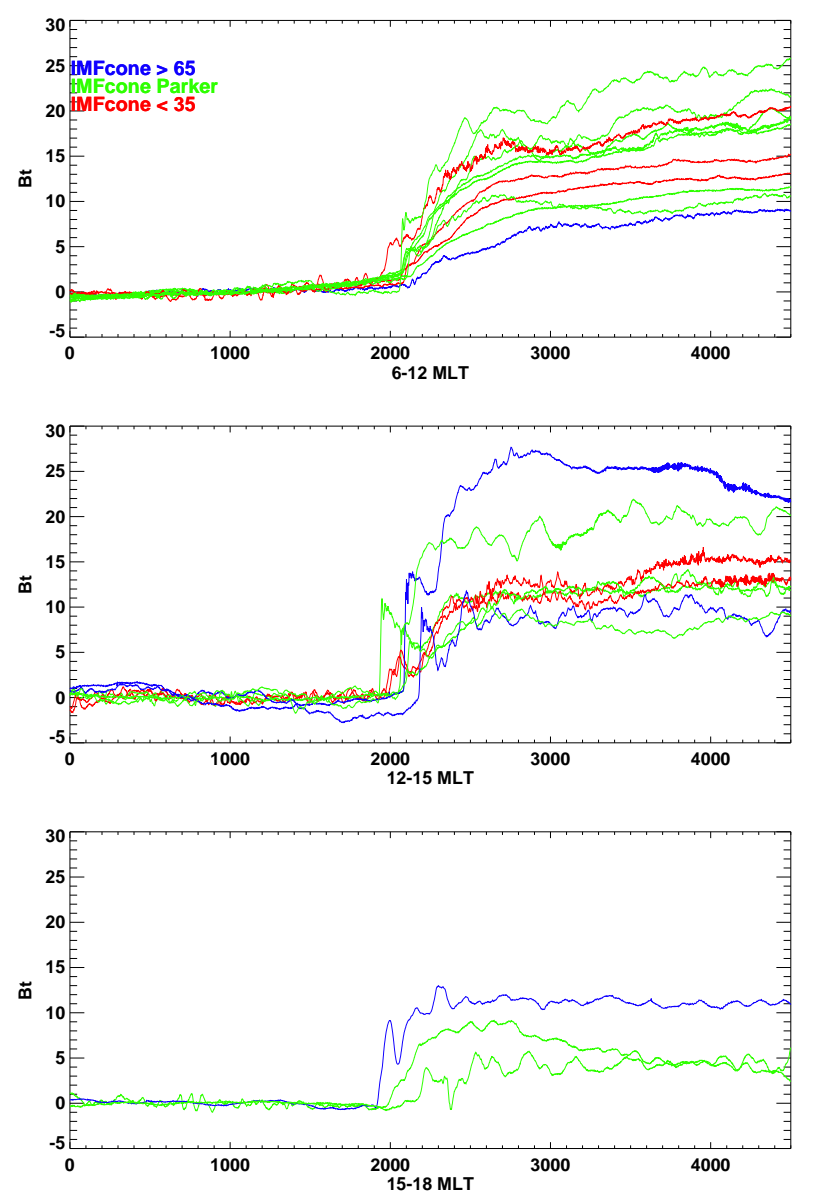

Figure 7. GOES spacecraft observations of the total magnetic field jump for 24 events in our data set, organized according to MLT. The mean level of the total magnetic field before the IP shock response $\left(B_{\mathrm{t}}\right.$ in $\mathrm{nT}$ ) has been subtracted. First panel shows the observations in which the spacecraft were located between 6 to 12 MLT, the central panel between 12 and 15 MLT, and the last panel between 15 to 18 MLT. The scale of the $x$ axis is 1 point $=0.512 \mathrm{~s}$.

Analysis of the events that occurred around 13.5 MLT, in the central panel of Fig. 7, reveals a very short period of pulsations with an average period of about $\sim 2 \mathrm{~s}$ after the first magnetic field enhancement. Those pulsations (Anderson et al., 1992a, b) in such locations are generated by the EMIC instability close to the magnetic equatorial plane. The wave amplitude is on the order of $2 \mathrm{nT}$.

\section{Interpretation}

Our analyses of the GOES spacecraft observations show that the IP shock interaction with the Earth's magnetosphere results systematically in double-step magnetic field profiles in the dayside geostationary orbit. Previously, there have only been case studies of double-step structures (e.g., Andréeová et al., 2011) and it has not been clear which processes cause 


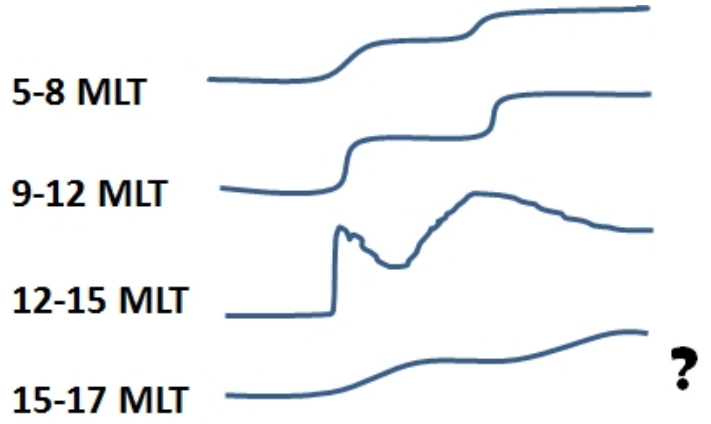

Figure 8. Sketch profiles of the total magnetic field observed in the geostationary orbit in four different sectors: $5-8,8-12,12-15$, and 15-17 MLT.

the double-step signature. In this paper, we reveal that the response propagates in the dayside magnetosphere at the average speed of $300-500 \mathrm{~km} \mathrm{~s}^{-1}$. The Alfvén speed in this region is considerably higher, on the order of $1500 \mathrm{~km} \mathrm{~s}^{-1}$ (estimated using THEMIS and LANL data), which implies that the response does not propagate in the Earth's magnetosphere as a compressional MHD wave. Chappell (1974) presented variation of the plasma density in the Earth's magnetosphere observed by the Ogo spacecraft. The typical density of the dayside magnetospheric plasma changes from the magnetopause up to the plasmapause, and in the geostationary orbit reaches in the average $0.1 \mathrm{~cm}^{-3}$. Laakso et al. (2002a, b) presented the density profiles within the magnetosphere recorded by the POLAR spacecraft. Samsonov et al. (2007) discussed MHD simulations, similar to those compared in the case study by Andréeová et al. (2011), where the density profiles in the dayside magnetosphere were overestimated, which was probably caused by the MHD approach. This allows us to suggest, based on the previous studies of the IP shock interaction with the bow shock (e.g., Šafránková et al., 2007; Juusola et al., 2010), that the multi-step feature in the geostationary magnetic field as a response to IP shock impact could be caused by the magnetopause oscillations.

Our study reveals that the local magnetic field depression observed by the GOES spacecraft peaks around 13.5 MLT, with a range from 12 to 15 MLT. LANL-94 probably observed the same ion population as the Cluster spacecraft inside the plasmasphere. We suggest that this MLT pattern on the GOES spacecraft is caused by pushing the plasmaspheric material over the geostationary orbit due to the magnetospheric compression and by the penetration of the magnetospheric convection. While the total magnetic field decreased after the first initial steep enhancement, the LANL94 spacecraft observed increase of the energetic ion density. The level of the energetic ions was comparable to that which was observed by Cluster-4, located on the edge of the plasmasphere. The location of the plasmaspheric bulge is also in good agreement with the previous observation (Chappell

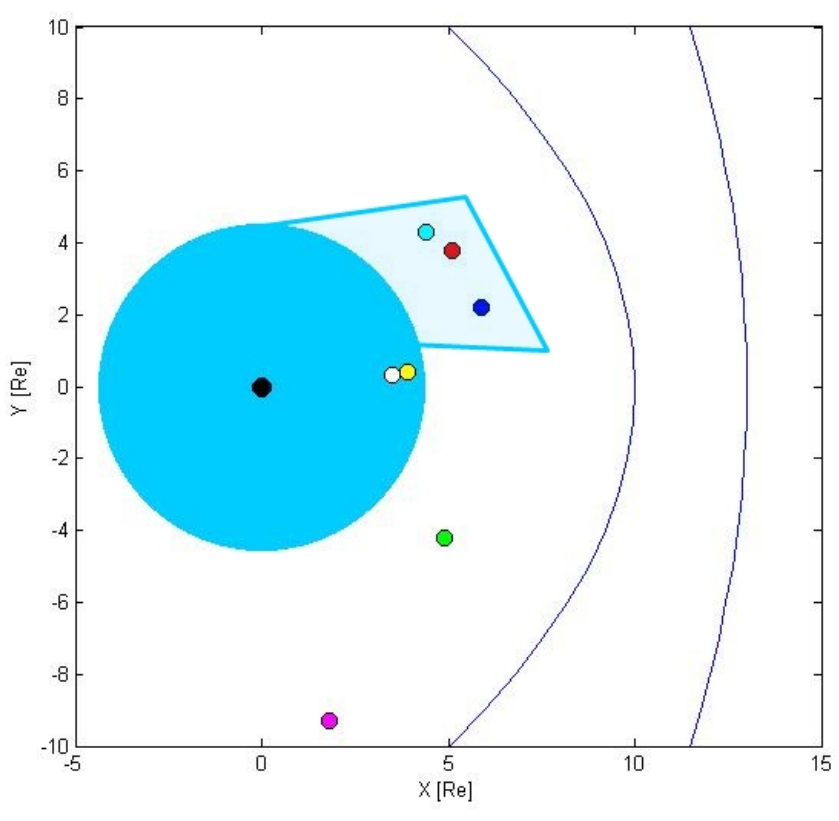

Figure 9. Sketch of the plasmaspheric bulge from the first event on 19 November 2007. The yellow and white dots denote the Cluster-4 and 2 spacecraft located in the edge of the plasmasphere, illustrated by the light blue circle. Red and blue dots represent GOES 10 and 12 locations within the plasmaspheric bulge, illustrated by the blue shaded area. The green dot represents GOES 11 spacecraft located in the dayside geostationary orbit outside the plasmaspheric bulge. The cyan dot shows the LANL-94 location and the magenta dot shows the THEMIS-D location. The bow shock and magnetopause are illustrated by the blue solid lines.

et al., 1971) of the low-energy ion transition locations in the outer region between 12-22 MLT.

The majority of events in the present study occurred during northward IMF, which led to a low magnetospheric activity. As was discussed by Elphic et al. (1996), there are two processes that determine how energetic ions from the plasmasphere can appear locally in the geostationary orbit. The first process assumes already existing ions in the orbit related to a plasmaspheric bulge intrusion driven by an earlier activity. For one of our case studies, we observed that after the magnetosphere was compressed, and the magnetospheric convection reversed (see the third panel in Fig. 1) and moved inward, bending the plasmaspheric bulge over the location of the GOES spacecraft in the 13.5 MLT. The second process assumes an initial quiet-time of almost circular plasmasphere without any bulge, due to the enhanced magnetospheric convection the plasmaspheric ions appeared in the geostationary orbit. Because the second process is very slow on the order of $6 \mathrm{~h}$, the more probable scenario turns out to be the already existing plasmaspheric bulge. Even if the convection is very weak, it is still an ongoing process in the Earth's magnetosphere, causing the skewness of the plasmasphere.

Figure 9 illustrates the possible scenario discussed above. 
Observation of EMIC waves after the first total magnetic field enhancement between $12-15$ in the geostationary orbit confirms the observation of the plasmaspheric bulge (Fuselier et al., 2004). Analysis of a case study of the 19 November 2007 event revealed an increase of the thermal anisotropy, while the total magnetic field decreased.

Figure 4 represents the time variation of the total ground magnetic field at low and mid latitudes. Ground magnetic field observations were supplemented by an already published double IP shock event on 9 November 2002. All ground magnetic field variations occurred around 1116 MLT, i.e., covering a wider MLT range when compared to that which was observed in the geostationary orbit. Because the coverage of the spacecraft in the geostationary orbit is limited maximally to two or three spacecraft, the magnetosphere-ionosphere coupling allows us to predict dayside geostationary observation and its asymmetry in the IP shock response. The different strength of the response visible in Fig. 7 depends on the IP shock conditions in the solar wind, e.g., plasma density compression ratio, magnetic field compression ratio, and solar wind speed.

Figure 7 displays different total magnetic field profiles according to MLT. The coloring highlights the IMF direction upstream (before) the IP shock in the solar wind. The blue color represents quasi-perpendicular direction of the IMF. The green color illustrates an almost Parker spiral IMF (4050 degrees), and the red color shows quasi-radial IMF conditions. The second panel shows observations from the GOES spacecraft almost behind the nose of the magnetopause. In the case of the radial IMF, most of the dayside magnetosphere is exposed to the influence of the foreshock. One of the expected results is the observation of the ULF wave activity in the dayside geostationary orbit (see Fig. 1). As the result of the ULF activity in the dayside magnetosphere, the IP shock response turns out to be smoothed and less steep around 13.5 MLT. The amplitude of the overall magnetic compression seems to be unaffected.

We did not find any dependence of the magnetospheric compression or the location of the total magnetic field decrease in the magnetosphere on the IMF direction upstream from the IP shock in the solar wind (see Fig. 7). Table 1 summarizes basic IP shock properties, such as the IP shock normal. Most of the IP shock had an almost parallel normal vector to the Sun-Earth line. Thus, with this data set, we cannot conclude any effect of the IP shock inclination effect on the Earth's magnetosphere. Other solar wind statistical properties will be discussed in a following work.

\section{Summary and conclusion}

In this paper, we have studied the response of magnetospheric magnetic fields to IP shocks using a wide MLT coverage of GOES spacecraft in the geostationary orbit. We analyzed 24 IP shocks, summarized in Table 1, and presented two of them in detail (19 and 12 November 2007).

The single abrupt change of all solar wind parameters and magnetic fields observed in the solar wind has a different response in different sectors of the dayside geostationary orbit, roughly sketched in Fig. 8. In the geostationary orbit, the IP shock response showed in all dayside cases a double-step profile of the magnetic field increase. Detailed geostationary observations revealed a clear asymmetry in the response profiles. The peak was strongest around 13.5 MLT, and the total magnetic field double-step profile revealed a magnetic field depression between the magnetic field enhancements. At other dayside MLT locations of the geostationary orbit, the initial response was smoother and slower, and no decrease was observed before the second step.

Low- and mid-latitude ground magnetic field observations were supplemented by the double IP shock event on 9 November 2002. The ground measurements revealed similar magnetic variation over the MLT to that which was observed in the geostationary orbit. Around 13.5 MLT the time derivatives of the magnetic field depict the peak.

We suggest that the asymmetry of the double-step structures are caused by the expansion of plasmaspheric ions to the geostationary orbit, caused by the magnetospheric compression due to the IP shock impact, leading to the plasmaspheric bulge bending over the spacecraft location around 13.5 MLT. We have observed Pc 1-2 pulsations with a period of about $\sim 2 \mathrm{~s}$, generated by the EMIC instability probably caused by the plasmaspheric bulge due to the thermal anisotropy.

ULF Pc3 wave activity may modify the IP shock response in the dayside magnetosphere, especially the steepness of the first magnetic field enhancement. We assume that ULF wave activity observed in the geostationary orbit resulted from the field line resonance. Compressional Pc3 pulsations, related to the wave-particle interaction in the foreshock and IP shock, are possible drivers.

Acknowledgements. This work was supported by the Academy of Finland. We thank the CDAWeb service and the corresponding PIs for the satellite data. We especially thank H. J. Singer for the high time-resolution GOES data.

Topical Editor C. Owen thanks two anonymous referees their help in evaluating this paper.

\section{References}

Anderson, B. J., Erlandson, R. E., and Zanetti, L. J.: A statistical study of Pc 1-2 magnetic pulsations in the equatorial magnetosphere. I - Equatorial occurrence distributions. II - Wave properties, J. Geophys. Res., 97, 3075-3101, doi:10.1029/91JA02706, 1992a.

Anderson, B. J., Erlandson, R. E., and Zanetti, L. J.: A statistical study of Pc 1-2 magnetic pulsations in the equatorial magne- 
tosphere 2. Wave properties, J. Geophys. Res., 97, 3089-3101, doi:10.1029/91JA02697, 1992b.

Andréeová, K.: The study of instabilities in the solar wind and magnetosheath and their interaction with the Earth's magnetosphere, Planet. Space Sci., 57, 888-890, doi:10.1016/j.pss.2008.12.005, 2009.

Andréeová, K., Pulkkinen, T. I., Laitinen, T. V., and Přech, L.: Shock propagation in the magnetosphere: Observations and MHD simulations compared, J. Geophys. Res., 113, A09224, doi:10.1029/2008JA013350, 2008.

Andréeová, K., Pulkkinen, T. I., Juusola, L., Palmroth, M., and Santolík, O.: Propagation of a shock-related disturbance in the Earth's magnetosphere, J. Geophys. Res.-Space Phys., 116, A01213, doi:10.1029/2010JA015908, 2011.

Angelopoulos, V.: The THEMIS Mission, Space Sci. Rev., 141, 534, doi:10.1007/s11214-008-9336-1, 2008.

Araki, T.: A Physical Model of the Geomagnetic Sudden Commencement, in: Solar Wind Sources of Magnetospheric UltraLow-Frequency Waves, edited by: Engebretson, M. J., Takahashi, K., and Scholer, M., 183 pp., 1994.

Balogh, A., Dunlop, M. W., Cowley, S. W. H., Southwood, D. J., Thomlinson, J. G., Glassmeier, K. H., Musmann, G., Luhr, H., Buchert, S., Acuna, M. H., Fairfield, D. H., Slavin, J. A., Riedler, W., Schwingenschuh, K., and Kivelson, M. G.: The Cluster Magnetic Field Investigation, Space Sci. Rev., 79, 65-91, 1997.

Chappell, C. R.: Recent satellite measurements of the morphology and dynamics of the plasmasphere., Rev. Geophys. Space Phys., 10, 951-979, doi:10.1029/RG010i004p00951, 1972.

Chappell, C. R.: Detached plasma regions in the magnetosphere, J. Geophys. Res., 79, 1861, doi:10.1029/JA079i013p01861, 1974.

Chappell, C. R., Harris, K. K., and Sharp, G. W.: The dayside of the plasmasphere, J. Geophys. Res., 76, 7632, doi:10.1029/JA076i031p07632, 1971.

Elphic, R. C., Weiss, L. A., Thomsen, M. F., McComas, D. J., and Moldwin, M. B.: Evolution of plasmaspheric ions at geosynchronous orbit during times of high geomagnetic activity, Geophys. Res. Lett., 23, 2189-2192, doi:10.1029/96GL02085, 1996.

Fairfield, D. H., Baumjohann, W., Paschmann, G., Luehr, H., and Sibeck, D. G.: Upstream pressure variations associated with the bow shock and their effects on the magnetosphere, J. Geophys. Res., 95, 3773-3786, doi:10.1029/JA095iA04p03773, 1990.

Fuselier, S. A., Gary, S. P., Thomsen, M. F., Claflin, E. S., Hubert, B., Sandel, B. R., and Immel, T.: Generation of transient dayside subauroral proton precipitation, J. Geophys. Res., 109, A12227, doi:10.1029/2004JA010393, 2004.

Grib, S. A.: Interaction of non-perpendicular/parallel solar wind shock waves with the Earth's magnetosphere, Space Sci. Rev., 32, 43-48, 1982.

Grib, S. A., Briunelli, B. E., Dryer, M., and Shen, W.-W.: Interaction of interplanetary shock waves with the bow shock-magnetopause system, J. Geophys. Res., 84, 5907-5921, 1979.

Juusola, L., Andréeová, K., Amm, O., Kauristie, K., Milan, S. E., Palmroth, M., and Partamies, N.: Effects of a solar wind dynamic pressure increase in the magnetosphere and in the ionosphere, Ann. Geophys., 28, 1945-1959, doi:10.5194/angeo-281945-2010, 2010.

Laakso, H., Pfaff, R., and Janhunen, P.: Polar observations of electron density distribution in the Earth's magnetosphere. 1. Statis- tical results, Ann. Geophys., 20, 1711-1724, doi:10.5194/angeo20-1711-2002, 2002a.

Laakso, H., Pfaff, R., and Janhunen, P.: Polar observations of electron density distribution in the Earth's magnetosphere. 2. Density profiles, Ann. Geophys., 20, 1725-1735, doi:10.5194/angeo-201725-2002, 2002b.

Lepping, R. P., Acũna, M. H., Burlaga, L. F., Farrell, W. M., Slavin, J. A., Schatten, K. H., Mariani, F., Ness, N. F., Neubauer, F. M., Whang, Y. C., Byrnes, J. B., Kennon, R. S., Panetta, P. V., Scheifele, J., and Worley, E. M.: The Wind Magnetic Field Investigation, Space Sci. Rev., 71, 207-229, 1995.

Lin, R. P., Anderson, K. A., Ashford, S., Carlson, C., Curtis, D., Ergun, R., Larson, D., McFadden, J., McCarthy, M., Parks, G. K., Rème, H., Bosqued, J. M., Coutelier, J., Cotin, F., D’Uston, C., Wenzel, K.-P., Sanderson, T. R., Henrion, J., Ronnet, J. C., and Paschmann, G.: A Three-Dimensional Plasma and Energetic Particle Investigation for the Wind Spacecraft, Space Sci. Rev., 71, 125-153, 1995.

McComas, D. J., Bame, S. J., Barraclough, B. L., Donart, J. R., Elphic, R. C., Gosling, J. T., Moldwin, M. B., Moore, K. R., and Thomsen, M. F.: Magnetospheric plasma analyzer - Initial threespacecraft observations from geosynchronous orbit, J. Geophys. Res., 98, 13 453, doi:10.1029/93JA00726, 1993.

McComas, D. J., Bame, S. J., Barker, P., Feldman, W. C., Phillips, J. L., Riley, P., and Griffee, J. W.: Solar Wind Electron Proton Alpha Monitor (SWEPAM) for the Advanced Composition Explorer, Space Sci. Rev., 86, 563-612, 1998.

McFadden, J. P., Carlson, C. W., Larson, D., Ludlam, M., Abiad, R., Elliott, B., Turin, P., Marckwordt, M., and Angelopoulos, V.: The THEMIS ESA Plasma Instrument and In-flight Calibration, Space Sci. Rev., 141, 277-302, doi:10.1007/s11214-008-9440-2, 2008.

Ogilvie, K. W., Chornay, D. J., Fritzenreiter, R. J., Hunsaker, F., Keller, J., Lobell, J., Miller, G., Scudder, J. D., Sittler, Jr., E. C., Torbert, R. B., Bodet, D., Needell, G., Lazarus, A. J., Steinberg, J. T., Tappan, J. H., Mavretic, A., and Gergin, E.: SWE, A Comprehensive Plasma Instrument for the Wind Spacecraft, Space Sci. Rev, 71, 55-77, 1995.

Prech, L., Safrankova, J., and Nemecek, Z.: Response of magnetospheric boundaries to the interplanetary shock: Themis contribution, Geophys. Res. Lett., 35, L17S02, doi:10.1029/2008GL033593, 2008.

Reme, H., Bosqued, J. M., Sauvaud, J. A., Cros, A., Dandouras, J., Aoustin, C., Bouyssou, J., Camus, T., Cuvilo, J., Martz, C., Medale, J. L., Perrier, H., Romefort, D., Rouzaud, J., D‘Uston, C., Mobius, E., Crocker, K., Granoff, M., Kistler, L. M., Popecki, M., Hovestadt, D., Klecker, B., Paschmann, G., Scholer, M., Carlson, C. W., Curtis, D. W., Lin, R. P., McFadden, J. P., Formisano, V., Amata, E., Bavassano-Cattaneo, M. B., Baldetti, P., Belluci, G., Bruno, R., Chionchio, G., di Lellis, A., Shelley, E. G., Ghielmetti, A. G., Lennartsson, W., Korth, A., Rosenbauer, H., Lundin, R., Olsen, S., Parks, G. K., McCarthy, M., and Balsiger, H.: The Cluster Ion Spectrometry (cis) Experiment, Space Sci. Rev., 79, 303-350, 1997.

Samsonov, A. A., Sibeck, D. G., and Imber, J.: MHD simulation for the interaction of an interplanetary shock with the Earth's magnetosphere, J. Geophys. Res., 112, A12220, doi:10.1029/2007JA012627, 2007. 
Singer, H. J., Matheson, L., Grubb, R., Newman, A., and Bouwer, S. D.: Monitoring space weather with the GEOS magnetometers, SPIE conference proceedings, GEOS-8 and Beyond, 2812, 299308, 1996.

Smith, C. W., L'Heureux, J., Ness, N. F., Acuña, M. H., Burlaga, L. F., and Scheifele, J.: The ACE Magnetic Fields Experiment, Space Sci. Rev., 86, 613-632, 1998.

Spreiter, J. R. and Stahara, S. S.: Gasdynamic and magnetohydrodynamic modeling of the magnetosheath: A tutorial, Adv. Space Res., 14, 5-19, 1994.

Šafránková, J., Němeček, Z., Přech, L., Samsonov, A. A., Koval, A., and Andréeová, K.: Interaction of interplanetary shocks with the bow shock, Planet. Space Sci., 55, 2324-2329, doi:10.1016/j.pss.2007.05.012, 2007.

Yue, C., Zong, Q. G., Zhang, H., Wang, Y. F., Yuan, C. J., Pu, Z. Y., Fu, S. Y., Lui, A. T. Y., Yang, B., and Wang, C. R.: Geomagnetic activity triggered by interplanetary shocks, J. Geophys. Res., 115, A00I05, doi:10.1029/2010JA015356, 2010.
Zhang, H., Sibeck, D. G., Zong, Q.-G., McFadden, J. P., Larson, D., Glassmeier, K.-H., and Angelopoulos, V.: Global magnetospheric response to an interplanetary shock: THEMIS observations, Ann. Geophys., 30, 379-387, doi:10.5194/angeo-30-3792012, 2012.

Zhang, X. Y., Zong, Q.-G., Wang, Y. F., Zhang, H., Xie, L., Fu, S. Y., Yuan, C. J., Yue, C., Yang, B., and Pu, Z. Y.: ULF waves excited by negative/positive solar wind dynamic pressure impulses at geosynchronous orbit, J. Geophys. Res., 115, A10221, doi:10.1029/2009JA015016, 2010.

Zhuang, H. C., Russell, C. T., Smith, E. J., and Gosling, J. T.: Threedimensional interaction of interplanetary shock waves with the bow shock and magnetopause - A comparison of theory with ISEE observations, J. Geophys. Res., 86, 5590-5600, 1981. 